\title{
Some remarks on segregation of $k$ species in strongly competing systems
}

\author{
FLAVIA LANZARA \\ Dipartimento di Matematica G. Castelnuovo, \\ Sapienza Università di Roma, \\ piazzale A. Moro 5, 00185 Roma, Italy \\ E-mail: flavia.lanzara@uniroma1.it \\ Eugenio Montefusco \\ Dipartimento di Matematica G. Castelnuovo, \\ Sapienza Università di Roma, \\ piazalle A. Moro 5, 00185 Roma, Italy \\ E-mail: eugenio.montefusco@uniroma1.it
}

[Received 11 May 2020 and in revised form 29 September 2020]

Spatial segregation occurs in population dynamics when $k$ species interact in a highly competitive way. As a model for the study of this phenomenon, we consider the competition-diffusion system of $k$ differential equations

$$
-\Delta u_{i}(x)=-\mu u_{i}(x) \sum_{j \neq i} u_{j}(x), \quad i=1, \ldots, k
$$

in a domain $D$ with appropriate boundary conditions. Any $u_{i}$ represents a population density and the parameter $\mu$ determines the interaction strength between the populations. The purpose of this paper is to study the geometry of the limiting configuration as $\mu \rightarrow+\infty$ on a planar domain for any number of species. If $k$ is even we show that some limiting configurations are strictly connected to the solution of a Dirichlet problem for the Laplace equation.

2020 Mathematics Subject Classification: Primary 35Bxx, 35J47; Secondary 92D25.

Keywords: Pattern formation, spatial segregation, strong competition.

\section{Introduction and setting of the problem}

When two or more species live in proximity and share the same basic requirements, they usually compete for resources, habitat or territory. Only the strongest prevails, driving the weaker competitors to extinction. This is the principle of competitive exclusion (also known as Gause's law). One species wins because its members are more efficient at finding resources, which leads to an increase in population. This means that a population of competitors finds less of the same resources and cannot grow at its maximal capacity [13].

According to the competitive exclusion principle, many competing species cannot coexist under very strong competition, but when spatial movements are permitted more than one species can coexist thanks to the segregation of their habitats. For a theoretical discussion and some experimental results see $[14,17]$. 
From a mathematical viewpoint the determination of the configuration of the habitat segregation for some populations is an interesting problem which can be modelled by an optimal (in a suitable sense) partition of a domain; for example in the papers [8-11,22] the problem is studied modelling the interspecies competition with a large interaction term in an elliptic system of partial differential equations inspired by classical models in populations dynamics. In [3, 4, 12] the problem is modelled as a Cauchy problem for a parabolic system of semilinear partial differential equations describing the dynamics of the densities of different species. In the evolutive case, in particular see [12], it is proved that some populations can vanish under the competition of other species; moreover, in [3, 4] the authors are able to estimate the number of the long-term surviving populations and other interesting qualitative properties of the spatial distributions of interacting populations. Note that also the study of the territoriality, that is how different groups of the same species divide an area, avoiding to effectively fight for resources, can be viewed as a habitat segregation produced by competition (see, for example, [5, 16, 20]); moreover, this kind of competition is a struggle between competitors having the same features, that is between perfect competitors.

Others approaches have been considered. In [6], for example, a phase segregation problem is studied by minimization of integral functionals. The authors obtain results showing mixing or separation of the two phases, depending of the strength of the interaction between the two species (that is depending on some relations between the parameters in the model). The main difference with our study is that, in [6], the densities have no to satisfy a boundary datum, so that the optimal partition of the domain is related only to the geometry of the domain and the interaction between the parameters.

As a model for the study of the segregation phenomena, we consider the competition-diffusion system of $k$ differential equations

$$
\left\{\begin{array}{ll}
-\Delta u_{i}(x)=-\mu u_{i}(x) \sum_{j \neq i} u_{j}(x) & \text { in } D, \\
u_{i}(x) \geqslant 0 & \text { in } D, \\
u_{i}(x)=\phi_{i}(x) & \text { on } \partial D .
\end{array} \quad i=1, \ldots, k .\right.
$$

Here $D \subseteq \mathbf{R}^{n}$ is an open bounded, simply connected domain with smooth boundary $\partial D$. In this paper we consider the case $n=2$. We assume that the function $\Phi=\left(\phi_{1}, \ldots, \phi_{k}\right)$ is an admissible datum that is $\phi_{i} \in W^{1, \infty}(\partial D), \phi_{i} \geqslant 0, i=1, \ldots, k, \phi_{i} \cdot \phi_{j}=0$ a.e. on $\partial D$ for $i \neq j$, the sets $\left\{\phi_{i}>0\right\}$ are nonempty, open connected arcs and the function $\sum_{i=1}^{k} \phi_{i}$ vanishes exactly in $k$ points of $\partial D$ (the endpoints of the $\phi_{i}$ 's supports).

The system (1.1) governs the steady states of $k$ competing species coexisting in the same domain $D$. Any $u_{i}$ represents a population density and the parameter $\mu>0$ determines the interaction strength between the populations. In this model the competition between two species is depicted without direct reference to the resources they share, rather, it is assumed that the presence of each population leads to a depression of its competitor's growth rate.

If $\Phi$ is admissible, the existence of positive solutions of (1.1) for any positive $\mu$ is proved in [9] using Leray-Schauder degree theory. The uniqueness is proved in [24], using the sub- and super-solution method. 
Let us define the class of segregated densities

$$
\begin{array}{ll}
u_{\Phi}=\left\{U=\left(u_{1}, \ldots, u_{k}\right) \in\left(H^{1}(D)\right)^{k}:\right. & u_{i}=\phi_{i} \text { on } \partial D \\
& u_{i} \geqslant 0 \text { in } D \\
& \left.u_{i} \cdot u_{j}=0 \text { for } i \neq j \text { a.e. in } D\right\}
\end{array}
$$

and the class

$$
\begin{aligned}
\delta_{\Phi}=\left\{U=\left(u_{1}, \ldots, u_{k}\right) \in U_{\Phi}:\right. & -\Delta u_{i} \leqslant 0 \text { in } D, \\
& \left.-\Delta\left(u_{i}-\sum_{j \neq i} u_{j}\right) \geqslant 0 \text { in } D\right\} .
\end{aligned}
$$

Let $U^{(\mu)}=\left(u_{1, \mu}, \ldots, u_{k, \mu}\right)$ be the solution of (1.1) for every $\mu>0$. In [9] it is proved that there exists $\bar{U}=\left(\bar{u}_{1}, \ldots, \bar{u}_{k}\right) \in U$ such that, up to subsequences, $u_{i, \mu} \rightarrow \bar{u}_{i}$ in $H^{1}(D)$ and $\delta_{\Phi}$ contains all the asymptotic limits of (1.1) that is $\bar{U} \in S_{\Phi}$.

The uniqueness of the limit solution of (1.1) as $\mu \rightarrow+\infty$ was proved in [9] in the case $k=2$ and in [11] in the case of $k=3$ and in dimension $n=2$. Specifically, the authors prove that the class $\delta_{\Phi}$ consists of one element. In [24] it is proved that $S_{\Phi}$ consists of one element also in the case of arbitrary dimension and arbitrary number of species. A different proof of uniqueness of the limit configuration, based on the maximum principle and on the qualitative properties of the elements of $\delta_{\Phi}$, is given in [2].

The description of the qualitative properties of the limiting configurations in the planar case (i.e., $n=2$ ) was considered in [11] for $k=3$ and in [19] for $k=4$. The aim of this paper is to describe the geometry of the limiting configurations in the planar case, for any number of species.

The outline of the paper is as follows. In Section 2 we recall some basic facts and known results of the class $\delta_{\Phi}$, which will be used later. In Section 3 we study the geometry of the limiting configuration in any number of species. If $k=2 s$, starting from the argument used in [19] for $k=4$ species, we prove that some limiting configurations are strictly related to the solution of a Dirichlet problem for the Laplace equation. Our results rely on the construction of a harmonic function which assumes the value $\sum_{j=1}^{2 s}(-1)^{j} \phi_{j}$ on $\partial D$. This function has an even number of nodal regions compatible with an alternate sign rule. We emphasize that this construction cannot be done in the case of odd $k$. This will be the object of a forthcoming paper. In Section 4 we focus on the case of $k=6$ number of species.

\section{Basic facts}

In this section we recall some basic facts that will play an important role in our study. Suppose that $D$ is a simply connected domain in $\mathbf{R}^{2}$. Due to the conformal invariance of the problem, with no loss of generality we can assume

$$
D=B(O, 1)=\left\{x=\left(x_{1}, x_{2}\right) \in \mathbf{R}^{2}:|x|<1\right\}
$$

and consider the class

$$
\begin{aligned}
\delta_{\Phi}= & \left\{U=\left(u_{1}, \ldots, u_{k}\right) \in\left(H^{1}(D)\right)^{k}: u_{i} \geqslant 0 \text { in } D, u_{i}=\phi_{i} \text { on } \partial D,\right. \\
& \left.u_{i} \cdot u_{j}=0 \text { for } i \neq j,-\Delta u_{i} \leqslant 0,-\Delta\left(u_{i}-\sum_{j \neq i} u_{j}\right) \geqslant 0 \text { in } D\right\} .
\end{aligned}
$$


The study of $S_{\Phi}$ provides the understanding of the segregated states of $k$ species induced by strong competition. If $\Phi=\left(\phi_{1}, \ldots, \phi_{k}\right)$ is an admissible datum then $\phi_{i}, i=1, \ldots, k$, are positive in their supports, the sets $\left\{\phi_{i}>0\right\} \subset \partial D$ are open connected arcs and $\Phi=\sum_{i=1}^{k} \phi_{i}$ vanishes at exactly $k$ points of $\partial D$, the endpoints $p_{1}, \ldots, p_{k}$ in counter clockwise order.

In the following we will denote by $U$ both the $k$-tuple $\left(u_{1}, \ldots, u_{k}\right)$ and the function $\sum_{i=1}^{k} u_{i}$. For any $U \in S_{\Phi}$ define the nodal regions

$$
\omega_{i}=\left\{p \in D: u_{i}(p)>0\right\} \quad i=1, \ldots, k,
$$

the multiplicity of a point $p \in \bar{D}$ with respect to $U$ :

$$
m(p)=\#\left\{i:\left|\omega_{i} \cap B_{r}(p)\right|>0 \quad \forall r>0\right\}
$$

where $B_{r}(p)=\left\{q \in \mathbf{R}^{2}:|p-q|<r\right\}$, and the interfaces between two densities

$$
\Gamma_{i j}=\partial \omega_{i} \cap \partial \omega_{j} \cap\{p \in D: m(p)=2\} .
$$

The element $U \in S_{\Phi}$ defines exactly $k$ nodal regions and

$$
\bar{D}=\bar{\omega}_{1} \cup \ldots \cup \bar{\omega}_{k} .
$$

Let us summarize the basic properties of the elements $U \in \mathcal{S}_{\Phi}$ :

(s1) Each $u_{i} \in W^{1, \infty}(\bar{D})$ (see [10, Theorem 8.4]). It follows that $u_{i} \in C(\bar{D}), \omega_{i}$ is open and $p \in \omega_{i}$ implies $m(p)=1$. By standard regularity theory for elliptic equations we also have that $u_{i} \in C^{\infty}\left(\omega_{i}\right)$;

(s2) each $\omega_{i}$ is connected and each $\Gamma_{i j}$ is either empty or a connected arc starting from a point $p_{i} \in \partial D$ (see [11, Remark 2.1]);

(s3) $u_{i}$ is harmonic in $\omega_{i} ; u_{i}-u_{j}$ is harmonic in $D \backslash \cup_{h \neq i, j} \bar{\omega}_{h}$ (see [10, Proposition 6.3]);

(s4) if $p \in D$ satisfies $m(p)=2$, then (see [10, Remark 6.4])

$$
\lim _{\omega_{i} \ni q \rightarrow p} \nabla u_{i}(q)=-\lim _{\omega_{j} \ni q \rightarrow p} \nabla u_{j}(q)
$$

(s5) $U \in W^{1, \infty}(\bar{D})$ and if $p \in D$ satisfies $m(p)=2$, then

$$
|\nabla U(p)|=\lim _{\omega_{i} \ni q \rightarrow p}\left|\nabla u_{i}(q)\right|=\lim _{\omega_{j} \ni q \rightarrow p}\left|\nabla u_{j}(q)\right| \neq 0
$$

and the set $\{q: m(q)=2\}$ is locally a $C^{1}$-curve through $p$ ending either at points with higher multiplicity, or at the boundary $\partial D$ (see [10, Lemma 9.4]);

(s6) if $p \in D$ satisfies $m(p) \geqslant 3$, then $|\nabla U(q)| \rightarrow 0$, as $q \rightarrow p$ (see [10, Theorem 9.3]);

(s7) the set $\{p \in D: m(p) \geqslant 3\}$ consists of a finite number of points (see [10, Lemma 9.11]);

(s8) if $p \in D$ with $m(p)=h \geqslant 3$ then there exists $\theta_{0} \in(-\pi, \pi]$ such that

$$
U(r, \theta)=r^{h / 2}\left|\cos \left(\frac{h}{2}\left(\theta+\theta_{0}\right)\right)\right|+o\left(r^{h / 2}\right)
$$

as $r \rightarrow 0$, where $(r, \theta)$ is a system of polar coordinates around $p$ (see [10, Theorem 9.6]).

REMARK 2.1 The asymptotic formula (2.1) describes the behavior of $U$ in a neighborhood of a multiple point in $D$. As a consequence, at multiple point $U \in \delta_{\Phi}$ shares the angle in equal parts. This property does not hold true if $U \in \mathcal{S}_{\Phi}$ has a multiple point $p$ on the boundary $\partial D$. 


\section{Results on $\boldsymbol{k}$ species}

Let $U \in \mathcal{S}_{\Phi}$, we define the set of points of multiplicity greater than or equal to $h \in \mathbf{N}$

$$
Z_{h}(U)=\{p \in \bar{D}: m(p) \geqslant h\} .
$$

The set $Z_{h}(U)$ consists of a finite number of isolated points [10, Lemma 9.11 and Theorem 9.13].

Proposition 3.1 Let $k \geqslant 3$ and $U \in S_{\Phi}$, then $Z_{3}(U)$ is nonempty and does not contain points of multiplicity higher than $k$.

Proof. If $\mathcal{Z}_{3}(U)=\emptyset$ then the interfaces $\Gamma_{i j}$ between any two densities do not intersect in $\bar{D}$. The function $\Phi=\sum_{i=1}^{k} \phi_{i}$ vanishes in exactly $k$ points and any $\Gamma_{i j}$ links a point $p_{i} \in \partial D$ to a point $p_{j} \in \partial D, i \neq j$. Therefore, if $k$ is odd there exists at least a point $p_{\ell} \in \partial D$ which belongs to two interfaces. Then $p_{\ell} \in Z_{3}(U)$ and we get a contradiction.

If $k$ is even, since $\phi$ vanishes in exactly $k$ points, then there are only $k / 2+1$ interfaces which are nonempty, with endpoints on the boundary and do not intersect. This contradicts the fact that $U \in S_{\Phi}$ defines $k$ nodal regions. It also implies that $Z_{s}(U)=\emptyset$ for $s>k$.

Let $U \in S_{\Phi}$. We associate to any $p \in \mathcal{Z}_{3}(U)$ the number

$$
i(p)=m(p)-2 .
$$

Proposition 3.2 Let $k \geqslant 2$. The following relation holds:

$$
k-2=\sum_{p \in Z_{3}(U)} i(p)
$$

Proof. If $k=2$ then $Z_{3}(U)=\emptyset$ and (3.1) is trivially satisfied. Let $k \geqslant 3$. First of all we want to point out that the set $\left(\cup \bar{\Gamma}_{i j}\right) \cup \partial D$, that is the union of the interfaces between any two species and the boundary of the disk forms a planar, connected graph, whose vertices are the points in $Z_{3}(U)$ and the zeros of the boundary datum. From classical arguments in graph theory (essentially the Euler polyhedral formula, see for example [18, Theorem 1.5.2]) it follows that

$$
n-m+f=2
$$

where $n$ is the number of the vertices of the graph, $m$ the number of the edges and $f$ the number of the faces. In our case we have that $n=\sharp\left\{Z_{3}(U) \cap D\right\}+k$, that is the multiple points in the disk plus the $k$ zeros of the boundary datum, $m=\sharp\left\{\Gamma_{i j}\right\}+k$, the number of the $\operatorname{arcs} \Gamma_{i j} \neq \emptyset$ plus $k$ (the number of the $\operatorname{arcs}\left\{\phi_{i}>0\right\} \subseteq \partial D$ ), and $f$ is the number of the nodal regions $\omega_{i}$ plus 1 so that $f=k+1$. Then, it follows that

$$
m=n+f-2=2 k+\sharp\left\{Z_{3}(U) \cap D\right\}-1 .
$$

Now we want to point out that, for any $p \in Z_{3}(U) \cap D$, the number $m(p)$ corresponds to the number of the arcs $\Gamma_{i j}$ such that $p \in \bar{\Gamma}_{i j}$, whereas for the others vertices $p \in \partial D$ (the zeros of $\Phi$ on the boundary) it holds that $m(p)$ is the number of the arcs $\Gamma_{i j}$ such that $p \in \bar{\Gamma}_{i j}$ augmented of 1 . 
Then, recalling that for any vertex $p \in \partial D \backslash Z_{3}(U)$ we have $m(p)=2$, we can write that

$$
\begin{aligned}
\sum_{p \in \mathbb{Z}_{3}(U)} i(p) & =\sum_{p \in \mathcal{Z}_{3}(U)}[m(p)-2]=\sum_{p \in \mathcal{Z}_{3}(U) \cap D}[m(p)-2]+\sum_{p \text { vertices on } \partial D}[m(p)-2] \\
& =\sum_{p \in \mathcal{Z}_{3}(U) \cap D}\left[\sharp\left\{\Gamma_{i j}: p \in \bar{\Gamma}_{i j}\right\}-2\right]+\sum_{p \text { vertices on } \partial D}\left[\sharp\left\{\Gamma_{i j}: p \in \bar{\Gamma}_{i j}\right\}+1-2\right] \\
& =\sum_{\substack{p \text { vertices } \\
\text { of the graph }}}\left[\sharp\left\{\Gamma_{i j}: p \in \bar{\Gamma}_{i j}\right\}-2\right]+k
\end{aligned}
$$

since the vertices on the boundary are exactly $k$. Then, it follows that

$$
\begin{aligned}
\sum_{p \in \mathrm{Z}_{3}(U)} i(p) & =\sum_{\begin{array}{c}
p \text { vertices } \\
\text { of the graph }
\end{array}}\left[\sharp\left\{\Gamma_{i j}: p \in \bar{\Gamma}_{i j}\right\}-2\right]+k=\sum_{\begin{array}{c}
p \text { vertices } \\
\text { of the graph }
\end{array}}\left[\sharp\left\{\Gamma_{i j}: p \in \bar{\Gamma}_{i j}\right\}\right]-2 n+k \\
& =2(m-k)-2 n+k=2(f-2)-k=k-2
\end{aligned}
$$

since the edges are the union of the interfaces $\Gamma_{i j}$ and of the $k$ arcs $\left\{\phi_{i}>0\right\} \subseteq \partial D$, and summing over the vertices any edge is counted twice.

REMARK 3.3 Note that, following [18], the set $\Gamma=\cup \bar{\Gamma}_{i j}$, that is the union of the interfaces between two species, the zeros of the boundary datum $\Phi$ and the points in $Z_{3}(U)$ form a tree, since it is a planar, acyclic, connected graph. The zeros of $\Phi$ are the leaves and the multiple points in $Z_{3}(U)$ are the other vertices. From classical arguments in graph theory it follows that the number of the arcs composing $\Gamma$ is $\left(k+\#\left\{Z_{3}(U)\right\}-1\right)$.

REMARK 3.4 Let $k \geqslant 3$. We want to point out that identity (3.1) implies that

$$
1 \leqslant \#\left\{Z_{3}(U)\right\} \leqslant k-2 .
$$

In the following we assume that the number of species $k$ is even, that is $k=2 s, s>1$. Then we can define a harmonic function having opposite signs on adjacent nodal regions and strictly connected to $U \in S_{\Phi}$.

Consider the boundary value problem

$$
\begin{cases}-\Delta \psi=0 & \text { in } D \\ \psi=\phi & \text { on } \partial D .\end{cases}
$$

Proposition 3.5 Let $\psi_{a}$ be the solution of (3.2) with boundary datum $\Phi^{a}=\sum_{j=1}^{2 s}(-1)^{j} \phi_{j}$ and let $U \in S_{\Phi}$.

1. If $U=\left|\psi_{a}\right|$ then for any $p \in Z_{3}(U) \cap D$ we have that $m(p)$ is even.

2. If there exists $p \in Z_{3}(U) \cap D$ such that $m(p)$ is odd then $U \neq\left|\psi_{a}\right|$.

Proof. 1. Let $U=\left|\psi_{a}\right|$ and $p \in Z_{3}(U)$. If $p \in D$ then $U(p)=0$ and $\nabla U(p)=(0,0)$ ([10, Theorem 9.3]). It follows that $p$ is a critical point for $\psi_{a}$ at level 0 . By standard theory of harmonic functions the zero set of $\psi_{a}$ around a critical point at level 0 is made by (at least) 4 half-lines, meeting with equal angles. We infer that locally around $p$ the function $\psi_{a}$ defines $q$ nodal components with $q \geqslant 4$ and $q$ is even because $\psi_{a}$ has alternate positive or negative sign on adjacent sets. 
2. Let $p \in Z_{3}(U)$ such that $m(p)=q$ with odd $q$. If $p \in D$ and $U=\left|\psi_{a}\right|$ then $p$ is a critical point for $\psi_{a}$ at level 0 . We infer that locally around $p$ the function $\psi_{a}$ defines $q$ nodal region, and $q$ is odd. This is a contradiction because $\psi_{a}$ has alternate positive or negative sign on adjacent sets.

Theorem 3.6 Let $\Phi=\left(\phi_{1}, \ldots, \phi_{2 s}\right)$ be an admissible datum. The harmonic function $\psi_{a}$ which solves (3.2) with boundary datum $\Phi^{a}=\sum_{j=1}^{2 s}(-1)^{j} \phi_{j}$ possesses at most $s-1$ critical points $q$ in $D$ such that $\psi_{a}(q)=0$.

Proof. Since $\Phi$ is an admissible datum, the solution of (3.2) with boundary datum $\Phi^{a}$ vanishes at exactly $2 s$ points on $\partial D$, each arc $\left\{\phi_{j}>0\right\} \subset \partial D$ is connected and $\psi_{a}$ has different signs on adjacent arcs.

Since a harmonic function does not admit closed level lines, the set $\Gamma=\left\{x \in D: \psi_{a}(x)=0\right\}$ has no closed loop. We infer that $\psi_{a}$ has alternate positive or negative sign on $\rho$ sets, with $s+1 \leqslant$ $\rho \leqslant 2 s$ : the nodal components of $\psi_{a}$ (see Figure 1 in the case $k=6$ ). The zero set of the harmonic function $\psi_{a}$ around a critical point at level 0 is made by (at least) 4 half-lines, meeting with equal angles. We infer that locally around each critical point at level 0 the function $\psi_{a}$ defines $\ell$ nodal components with $4 \leqslant \ell \leqslant \rho$ and $\ell$ is even because $\psi_{a}$ has alternate positive or negative sign on adjacent sets.

Suppose that $\psi_{a}$ has $r$ critical points $q_{1}, \ldots, q_{r}$ in $D$ such that $\psi_{a}\left(q_{j}\right)=0, j=1, \ldots, r$, $r \geqslant 1$. Then $q_{j} \in Z_{3}\left(\left|\psi_{a}\right|\right)$ with $m\left(q_{j}\right) \geqslant 4, j=1, \ldots, r$. If $\psi_{a}$ defines $2 s$ nodal regions, i.e., $\rho=2 s$ then the function $U=\left|\psi_{a}\right|$ is an element of $S_{\Phi}$. From (3.1),

$$
2 s-2=\sum_{p \in \mathbb{Z}_{3}(U)} i(p) \geqslant \sum_{j=1}^{r} i\left(q_{j}\right)=\sum_{j=1}^{r} m\left(q_{j}\right)-2 r \geqslant 4 r-2 r=2 r
$$

that is $r \leqslant s-1$. If $\psi_{a}$ defines $\rho$ nodal regions with $\rho<2 s$ then, by repeating the same argument in the proof of formula (3.1), we obtain that

$$
2 \rho-2-2 s=\sum_{p \in \mathcal{Z}_{3}\left(\left|\psi_{a}\right|\right)} i(p)
$$

Then,

$$
2 \rho-2-2 s=\sum_{p \in \mathbb{Z}_{3}\left(\left|\psi_{a}\right|\right)} i(p) \geqslant \sum_{j=1}^{r} i\left(q_{j}\right)=\sum_{j=1}^{r} m\left(q_{j}\right)-2 r \geqslant 4 r-2 r=2 r .
$$

We infer that $r<\rho-s-1<s-1$.

If the solution $\psi_{a}$ to (3.2) with alternate sign on adjacent arcs has exactly $s-1$ critical points at level zero we can say much more.

Proposition 3.7 Let $\Phi=\left(\phi_{1}, \ldots, \phi_{2 s}\right)$ be an admissible boundary datum and suppose that the harmonic function $\psi_{a}$, solution to (3.2) with boundary datum $\Phi^{a}=\sum_{j=1}^{2 s}(-1)^{j} \phi_{j}$, has $q_{1}, \ldots, q_{s-1}$ critical points in $D$ such that $\psi_{a}\left(q_{i}\right)=0, i=1, \ldots, s-1$. Then $q_{1}, \ldots, q_{s-1}$ are 4-points for the function $U=\left|\psi_{a}\right| \in \mathcal{S}_{\Phi}$. 


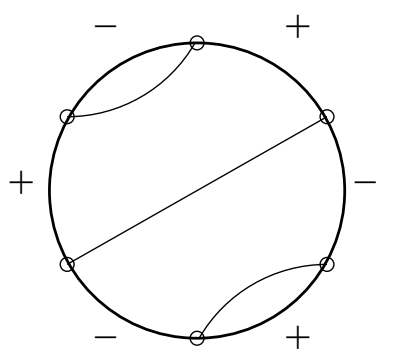

(a)

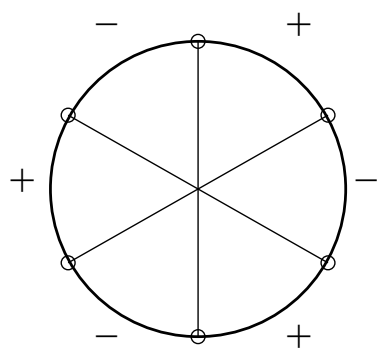

(c)

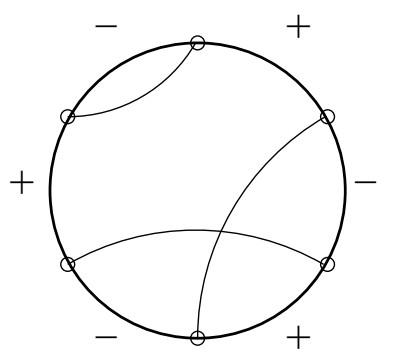

(b)

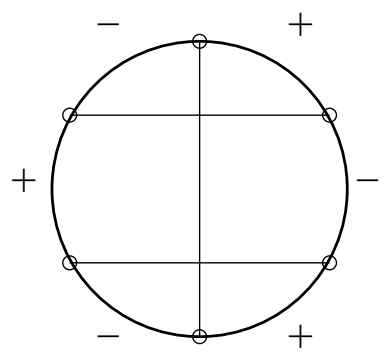

(d)

FIG. 1. The level set $\Gamma=\left\{x \in D: \psi_{a}(x)=0\right\}$ and the nodal components of $\psi_{a}$, where the function $\psi_{a}$ solves (3.2) with boundary datum $\Phi^{a}=\sum_{j=1}^{6}(-1)^{j} \phi_{j}$. Three different situations can occur: the function $\psi_{a}$ has 4 nodal regions (Figure a), 5 nodal regions (Figure b) or 6 nodal regions (Figures $\mathrm{c}$ and $\mathrm{d}$ ).

Proof. The zero set of the harmonic function $\psi_{a}$ around a critical point at level 0 is made by (at least) 4 half-lines, meeting with equal angles. We infer that locally around each $q_{i}, i=1, \ldots, s-1$, the function $\psi_{a}$ defines $\ell$ nodal components with $\ell \geqslant 4$. The function $\psi_{a}$ defines $\rho \leqslant 2 s$ nodal regions. From formula (3.3),

$$
2 \rho-2-2 s=\sum_{p \in Z_{3}\left(\left|\psi_{a}\right|\right)}(m(p)-2) \geqslant \sum_{j=1}^{s-1}\left(m\left(q_{j}\right)-2\right) \geqslant 2(s-1) .
$$

We infer that $\rho \geqslant 2 s$, hence $\rho=2 s$.

The function $U=\left|\psi_{a}\right|$ is nonnegative, satisfies the boundary datum and has exactly $2 s$ nodal regions. This function generates an element of $\delta_{\Phi}$, with boundary datum $\Phi$ and $\left\{q_{1}, \ldots, q_{s-1}\right\} \in$ $Z_{3}(U)$. Hence, we have

$$
\left\{q_{1}, \ldots, q_{s-1}\right\} \subseteq Z_{3}(U), \quad m\left(q_{j}\right)=m_{j}
$$

with $m_{j} \geqslant 4, j=1, \ldots, s-1$. From (3.1) we get

or equivalently

$$
\sum_{j=1}^{s-1} i\left(q_{j}\right) \leqslant 2 s-2
$$

$$
\sum_{j=1}^{s-1} m_{j}-2(s-1) \leqslant 2 s-2 \Longleftrightarrow \sum_{j=1}^{s-1} m_{j} \leqslant 4(s-1) .
$$

Since $m_{j} \geqslant 4$ we deduce that $m_{j}=4, j=1, \ldots, s-1$. 
Proposition 3.8 Let $\Phi=\left(\phi_{1}, \ldots, \phi_{2 s}\right)$ be an admissible datum. If $U \in \mathcal{S}_{\Phi}$ possesses a $2 s$-point $a_{U}$ in $\bar{D}$ then $U=\left|\psi_{a}\right|$, where $\psi_{a}$ is the harmonic function such that $\psi_{a}=\Phi^{a}=\sum_{j=1}^{2 s}(-1)^{j} \phi_{j}$ on $\partial D$. If $a_{U} \in D$ then $a_{U}$ is a critical point for $\psi_{a}$ at zero level.

Proof. Let $U=\left(u_{1}, \ldots, u_{2 s}\right)$ be an element of $\delta_{\Phi}$ with a $2 s$-point $a_{U}$, then there exist $2 s-1$ or $2 s$ arcs connecting $a_{U}$ to any of the isolated zeros of the boundary datum (note that there are $2 s-1$ arcs if and only if $\left.a_{U} \in \partial D\right)$. Then the function $\psi_{a}=\sum_{j=1}^{2 s}(-1)^{j} u_{j}$ is harmonic in $D \backslash$ $\left\{a_{U}\right\}$ (see [10, Proposition 6.3]), moreover, $\psi_{a}$ is bounded so, by Schwarz's removable singularity principle (see [21, Proposition 11.1]) $\psi_{a}$ is harmonic in $D$ and, by construction, $U=\left|\psi_{a}\right|$ in $D$ and $U=\left|\Phi^{a}\right|=\Phi$ on $\partial D$.

If $a_{U} \in \mathcal{Z}_{3}(U) \cap D$ then, for the property (s6), $U\left(a_{U}\right)=0$ and $\nabla U\left(a_{U}\right)=(0,0)$. Hence $\psi_{a}\left(a_{U}\right)=0$ and $\nabla \psi_{a}\left(a_{U}\right)=(0,0)$.

We can generalized Proposition 3.8 as follows.

Proposition 3.9 Let $\Phi=\left(\phi_{1}, \ldots, \phi_{2 s}\right)$ be an admissible datum. Let $U \in \mathcal{S}_{\Phi}$ be such that $Z_{3}(U)$ contains only points with even multiplicity in $\bar{D}$. Then $U=\left|\psi_{a}\right|$, where $\psi_{a}$ is the solution of the Dirichlet problem (3.2) with boundary datum $\psi_{a}=\Phi^{a}=\sum_{j=1}^{2 s}(-1)^{j} \phi_{j}$ on $\partial D$. Moreover, any $p \in Z_{3}(U) \cap D$ is a critical point for $\psi_{a}$ at zero level.

Proof. Let $U$ be an element of $\delta_{\Phi}$ such that $Z_{3}(U)=\left\{q_{1}, \ldots, q_{r}\right\}$ with $m\left(q_{i}\right)=2 \ell_{i} \geqslant 4$, $i=1, \ldots, r$. Then, for every $i=1, \ldots, r$, there exist $s_{1}^{(i)}, \ldots, s_{2 \ell_{i}}^{(i)} \in\{1, \ldots, 2 s\}$ such that

(i) $\bar{\omega}_{s_{1}^{(i)}} \cap \ldots \cap \bar{\omega}_{s_{2 \ell_{i}}^{(i)}}=\left\{q_{i}\right\}$;

(ii) $\sum_{j=1}^{2 \ell_{i}}(-1)^{j} u_{s_{j}}$ is harmonic in $\dot{\Omega}_{i} \backslash\left\{q_{i}\right\}$ where $\Omega_{i}=\bar{\omega}_{s_{1}^{(i)}} \cup \ldots \cup \bar{\omega}_{s_{2 \ell_{i}}}$ and $\dot{\Omega}_{i}$ denotes the interior of $\Omega_{i}$.

Keeping in mind that $u_{i}=0$ in $\omega_{j}, i \neq j$, then $\psi_{a}=\sum_{j=1}^{2 s}(-1)^{j} u_{j}$ is harmonic in $\dot{\Omega}_{i} \backslash\left\{q_{i}\right\}$, $i=1, \ldots, r$. It is easy to see that $\dot{\Omega}_{1} \cup \ldots \cup \dot{\Omega}_{r}=D \backslash\left\{q_{1}, \ldots, q_{r}\right\}$. Then the function $\psi_{a}=$ $\sum_{j=1}^{2 s}(-1)^{j} u_{j}$ is harmonic in $D \backslash\left\{q_{1}, \ldots, q_{r}\right\}$ (see [10, Proposition 6.3]), moreover, $\psi_{a}$ is bounded so, by Schwarz's removable singularity principle (see [21, Proposition 11.1]) $\psi_{a}$ is harmonic in $D$ and, by construction, $U=\left|\psi_{a}\right|$ in $D$ and $U=\left|\Phi^{a}\right|=\Phi$ on $\partial D$. $(0,0)$.

If $q \in Z_{3}(U) \cap D$ then $U(q)=0$ and $\nabla U(q)=(0,0)$. Hence $\psi_{a}(q)=0$ and $\nabla \psi_{a}(q)=$

The next Proposition gives conditions on the admissible datum $\Phi$ such that the limiting configuration has a point with multiplicity $2 s$ in $D$.

Proposition 3.10 Let $\Phi=\left(\phi_{1}, \ldots, \phi_{2 s}\right)$ be an admissible boundary datum and let $\psi_{a}$ be the solution to (3.2) with boundary datum $\Phi^{a}=\sum_{j=1}^{2 s}(-1)^{j} \phi_{j}$. Let $p \in D$ such that $\Phi^{a}$ satisfies the conditions

$$
\begin{gathered}
\int_{\partial D} \Phi^{a}\left(\frac{\zeta+p}{\bar{p} \zeta+1}\right) T_{j}\left(\zeta_{1}\right) d s_{\zeta}=0, \quad j=0,1, \ldots, s-1 \\
\int_{\partial D} \Phi^{a}\left(\frac{\zeta+p}{\bar{p} \zeta+1}\right) \zeta_{2} U_{j-1}\left(\zeta_{1}\right) d s_{\zeta}=0, \quad j=1, \ldots, s-1
\end{gathered}
$$


with $\zeta=\left(\zeta_{1}, \zeta_{2}\right)$, where $T_{j}$ and $U_{j}$ denote the Chebyshev polynomials of the first and second kind, respectively. Then $p$ is a 2 s-point for the function $U=\left|\psi_{a}\right| \in \mathcal{S}_{\Phi}$.

Proof. We introduce the transformation

$$
x=R_{p}(\zeta)=\frac{\zeta+p}{\bar{p} \zeta+1}
$$

Here we identify the complex numbers $x=x_{1}+i x_{2}$ and $\zeta=\zeta_{1}+i \zeta_{2}$ with the points $\left(x_{1}, x_{2}\right)$ and $\left(\zeta_{1}, \zeta_{2}\right) \in \mathbf{R}^{2}$, respectively. $R_{p}$ is a conformal map which maps the unit disk $\bar{D}$ into itself such that $R_{p}(\partial D)=\partial D$ and $R_{p}(0)=p$. Set

$$
\widetilde{\Psi}_{a}(\zeta)=\psi_{a}\left(R_{p}(\zeta)\right), \quad \widetilde{\Phi}^{a}(\zeta)=\Phi^{a}\left(R_{p}(\zeta)\right)
$$

Then $\widetilde{\Psi}_{a}$ solves the problem

$$
\begin{cases}-\Delta \widetilde{\Psi}_{a}=0 & \text { in } D \\ \widetilde{\Psi}_{a}=\widetilde{\Phi}^{a} & \text { on } \partial D\end{cases}
$$

Introducing a system of polar coordinates $(r, \theta)$, we can write the Fourier expansion of $\widetilde{\Psi}_{a}$

$$
\widetilde{\Psi}_{a}(r, \theta)=\frac{A_{0}}{2}+\sum_{j=1}^{\infty}\left(A_{j} \cos (j \theta)+B_{j} \sin (j \theta)\right) r^{j}, \quad \zeta=(r, \theta)
$$

Let $T_{j}$ and $U_{j}$ denote the Chebychev polynomials of the first and second kind, respectively. Keeping in mind the representation $T_{j}(\cos (\theta))=\cos (j \theta)$ and $U_{j-1}(\cos (\theta))=\sin (j \theta) / \sin (\theta)$ $([1,22.3 .15-16])$ from the conditions (3.4)-(3.5) we get

$$
\begin{aligned}
& A_{j}:=\frac{1}{\pi} \int_{-\pi}^{\pi} \widetilde{\Phi}^{a}\left(e^{i \theta}\right) \cos (j \theta) d \theta=0, \quad j=0,1, \ldots, s-1, \\
& B_{j}:=\frac{1}{\pi} \int_{-\pi}^{\pi} \widetilde{\Phi}^{a}\left(e^{i \theta}\right) \sin (j \theta) d \theta=0, \quad j=1, \ldots, s-1 .
\end{aligned}
$$

It follows that, around the origin,

$$
\widetilde{\Psi}_{a}(r, \theta)=\sum_{j=s}^{\infty}\left(A_{j} \cos (j \theta)+B_{j} \sin (j \theta)\right) r^{j}, \quad \zeta=(r, \theta) .
$$

We have $\left(A_{s}, B_{s}\right) \neq(0,0)$. Indeed, if not, let $\left(A_{v}, B_{v}\right) \neq(0,0)$ where $v>s$ is the index of the first nonzero Fourier component. Then there would be $2 v$ arcs starting form the origin, on which $\widetilde{\Psi}_{a}$ vanishes. Since a harmonic function does not admit closed level lines, this contradicts the fact that $\widetilde{\Phi}^{a}$ has exactly $2 s$ zeros.

Therefore $U(x)=\left|\psi_{a}(x)\right|=\left|\widetilde{\Psi}_{a}\left(R_{p}^{-1}(x)\right)\right|$ is nonnegative, satisfies the boundary datum $\Phi$ and has exactly $2 s$ nodal regions. This function generates an element of $\delta_{\Phi}$, with datum $\Phi$ and the $2 s$-point $p$ (see also [11, Lemma 3.2]).

Conversely, we have the following result. 
Proposition 3.11 Suppose that $\Phi=\left(\phi_{1}, \ldots, \phi_{2 s}\right)$ is an admissible datum and the function $U \in \mathcal{S}_{\Phi}$ generates a configuration with a $2 s$-point in $p \in D$. Then $\Phi^{a}=\sum_{j=1}^{2 s}(-1)^{j} \phi_{j}$ satisfies the conditions (3.4)-(3.5).

Proof. For Proposition 3.8, we have $U=\left|\psi_{a}\right|$ with $\psi_{a}$ solution of (3.2) with boundary datum $\Phi^{a}=\sum_{j=1}^{2 s}(-1)^{j} \phi_{j}$. Then the function $\widetilde{\Psi}_{a}(\zeta)=\psi_{a}\left(R_{p}(\zeta)\right)$, with $R_{p}$ in (3.6), solves (3.8), it is given by

$$
\widetilde{\Psi}_{a}(\zeta)=\frac{1-|\zeta|^{2}}{2 \pi} \int_{\partial D} \frac{\widetilde{\Phi}^{a}(\eta)}{|\zeta-\eta|^{2}} d s_{\eta}
$$

and it belongs to $C^{2}(D) \cap C^{0}(\bar{D})$ (cf. [15, (2.27)]).

On the other hand in the Fourier expansion (3.9) of $\widetilde{\Psi}_{a}$ we have

$$
A_{0}=A_{j}=B_{j}=0, \quad j=1, \ldots, s-1
$$

and $\left(A_{s}, B_{s}\right) \neq(0,0)$. The result follows from (3.10)-(3.11).

Proposition 3.12 Let $\Phi=\left(\phi_{1}, \ldots, \phi_{2 s}\right)$ be an admissible boundary datum, $\Phi^{a}=\sum_{j=1}^{2 s}(-1)^{j} \phi_{j}$ and $p \in D$. Conditions (3.4)-(3.5) are equivalent to

$$
\int_{\partial D} \Phi^{a}\left(\frac{\zeta+p}{\bar{p} \zeta+1}\right) \zeta_{1}^{j-h} \zeta_{2}^{h} d s_{\zeta}=0, \quad h=0, \ldots, j ; j=0, \ldots, s-1
$$

with $\zeta=\left(\zeta_{1}, \zeta_{2}\right)$.

Proof. From the representations

$$
\begin{aligned}
& \sin (j \theta)=\sum_{\substack{h=1 \\
h \text { odd }}}^{j}(-1)^{\frac{h-1}{2}}\left(\begin{array}{l}
j \\
h
\end{array}\right) \cos ^{j-h} \theta \sin ^{h} \theta, \\
& \cos (j \theta)=\sum_{\substack{h=0 \\
h \text { even }}}^{j}(-1)^{\frac{h}{2}}\left(\begin{array}{l}
j \\
h
\end{array}\right) \cos ^{j-h} \theta \sin ^{h} \theta
\end{aligned}
$$

we infer that, setting $\zeta_{1}=\cos (\theta), \zeta_{2}=\sin (\theta)$,

$$
\begin{gathered}
\zeta_{2} U_{j-1}\left(\zeta_{1}\right)=\sum_{\substack{h=1 \\
h \text { odd }}}^{j}(-1)^{\frac{h-1}{2}}\left(\begin{array}{l}
j \\
h
\end{array}\right) \zeta_{1}^{j-h} \zeta_{2}^{h}, \quad \zeta_{1}^{2}+\zeta_{2}^{2}=1, \quad j \geqslant 1 \\
T_{j}\left(\zeta_{1}\right)=\sum_{\substack{h=0 \\
h \text { even }}}^{j}(-1)^{\frac{h}{2}}\left(\begin{array}{l}
j \\
h
\end{array}\right) \zeta_{1}^{j-h} \zeta_{2}^{h}, \quad \zeta_{1}^{2}+\zeta_{2}^{2}=1, j \geqslant 0 .
\end{gathered}
$$

If conditions (3.13) are satisfied, from the representations (3.14)-(3.15), we deduce that even conditions (3.4)-(3.5) are.

Conversely, let conditions (3.4)-(3.5) hold. For $j=0$, since $T_{0} \equiv 1$, we get

$$
\int_{\partial D} \Phi^{a}\left(\frac{\zeta+p}{\bar{p} \zeta+1}\right) d s_{\zeta}=0 .
$$


For $j=1$, since $T_{1}\left(\zeta_{1}\right)=\zeta_{1}$ and $U_{0} \equiv 1$, we get

$$
\int_{\partial D} \Phi^{a}\left(\frac{\zeta+p}{\bar{p} \zeta+1}\right) \zeta_{1} d s_{\zeta}=\int_{\partial D} \Phi^{a}\left(\frac{\zeta+p}{\bar{p} \zeta+1}\right) \zeta_{2} d s_{\zeta}=0 .
$$

For $j=2$, from the relations $T_{2}\left(\zeta_{1}\right)=2 \zeta_{1}^{2}-1$ and $U_{1}\left(\zeta_{1}\right)=2 \zeta_{1}$, and (3.16) we get

$$
\begin{aligned}
& 0=\int_{\partial D} \Phi^{a}\left(\frac{\zeta+p}{\bar{p} \zeta+1}\right)\left(2 \zeta_{1}^{2}-1\right) d s_{\zeta}=2 \int_{\partial D} \Phi^{a}\left(\frac{\zeta+p}{\bar{p} \zeta+1}\right) \zeta_{1}^{2} d s_{\zeta}, \\
& 0=\int_{\partial D} \Phi^{a}\left(\frac{\zeta+p}{\bar{p} \zeta+1}\right) \zeta_{2}\left(2 \zeta_{1}\right) d s_{\zeta}=2 \int_{\partial D} \Phi^{a}\left(\frac{\zeta+p}{\bar{p} \zeta+1}\right) \zeta_{1} \zeta_{2} d s_{\zeta} .
\end{aligned}
$$

Since $\zeta_{1}^{2}+\zeta_{2}^{2}=1$, we also get

$$
0=\int_{\partial D} \Phi^{a}\left(\frac{\zeta+p}{\bar{p} \zeta+1}\right) \zeta_{2}^{2} d s_{\zeta}
$$

We proceed by induction on $j$. Suppose that conditions (3.13) are valid for $h=0, \ldots, j$ with $j<s-1$. We prove that

$$
\int_{\partial D} \Phi^{a}\left(\frac{\zeta+p}{\bar{p} \zeta+1}\right) \zeta_{1}^{j+1-h} \zeta_{2}^{h} d s_{\zeta}=0, \quad h=0, \ldots, j+1 .
$$

Using the inverse formula (see [7, p. 412])

$$
\zeta_{1}^{j+1}=2^{-j} \sum_{\substack{i=0 \\
j+1-i \text { even }}}^{j+1}\left(\begin{array}{c}
j+1 \\
i
\end{array}\right) T_{i}\left(\zeta_{1}\right), \quad j \geqslant 0
$$

where the prime at the sum symbol means that the first term (at $i=0$ ) is to be halved unless it is skipped, and conditions (3.4) we get

$$
\int_{\partial D} \Phi^{a}\left(\frac{\zeta+p}{\bar{p} \zeta+1}\right) \zeta_{1}^{j+1} d s_{\zeta}=0,
$$

that is (3.17) for $h=0$. If $h=2 \ell$ with $0<\ell \leqslant(j+1) / 2$, we have, keeping in mind (3.18) and (3.4),

$$
\begin{aligned}
\int_{\partial D} \Phi^{a}\left(\frac{\zeta+p}{\bar{p} \zeta+1}\right) \zeta_{1}^{j+1-2 \ell} \zeta_{2}^{2 \ell} d s_{\zeta} & =\int_{\partial D} \Phi^{a}\left(\frac{\zeta+p}{\bar{p} \zeta+1}\right) \zeta_{1}^{j+1-2 \ell}\left(1-\zeta_{1}^{2}\right)^{\ell} d s_{\zeta} \\
& =\sum_{i=0}^{\ell}\left(\begin{array}{l}
\ell \\
i
\end{array}\right)(-1)^{\ell-i} \int_{\partial D} \Phi^{a}\left(\frac{\zeta+p}{\bar{p} \zeta+1}\right) \zeta_{1}^{j+1-2 i} d s_{\zeta}=0
\end{aligned}
$$

If $h=2 \ell+1$, with $0 \leqslant \ell \leqslant j / 2$, we can write (3.17) as

$$
\int_{\partial D} \Phi^{a}\left(\frac{\zeta+p}{\bar{p} \zeta+1}\right) \zeta_{1}^{j-2 \ell} \zeta_{2}^{2 \ell+1} d s_{\zeta}=\sum_{i=0}^{\ell}\left(\begin{array}{l}
\ell \\
i
\end{array}\right)(-1)^{\ell-i} \int_{\partial D} \Phi^{a}\left(\frac{\zeta+p}{\bar{p} \zeta+1}\right) \zeta_{2} \zeta_{1}^{j-2 i} d s_{\zeta}
$$


From the relations $T_{0}\left(\zeta_{1}\right)=U_{0}\left(\zeta_{1}\right), T_{1}\left(\zeta_{1}\right)=2^{-1} U_{1}\left(\zeta_{1}\right), T_{\kappa}\left(\zeta_{1}\right)=2^{-1}\left(U_{\kappa}\left(\zeta_{1}\right)-U_{\kappa-2}\left(\zeta_{1}\right)\right)$, $\kappa \geqslant 2$, and the inverse formula (3.18), we infer that $\zeta_{1}^{j-2 i}$ can be expressed as a linear combination of the polynomials $U_{\kappa}\left(\zeta_{1}\right), 0 \leqslant \kappa \leqslant j-2 i$. Hence, conditions (3.5) imply that the integrals in the right-hand side of (3.19) vanish.

For example, if $k=4$ the conditions (3.13) reduce to

$$
\int_{\partial D} \Phi^{a}\left(\frac{\zeta+p}{\bar{p} \zeta+1}\right) d s_{\zeta}=0, \quad \int_{\partial D} \Phi^{a}\left(\frac{\zeta+p}{\bar{p} \zeta+1}\right) \zeta_{j} d s_{\zeta}=0, \quad j=1,2,
$$

which were obtained in [19, Proposition 3.12].

As a consequence of Propositions 3.10, 3.11 and 3.12 we deduce the following necessary and sufficient conditions such that $p \in D$ is a point with multiplicity $2 s$.

Theorem 3.13 Let $\Phi=\left(\phi_{1}, \ldots, \phi_{2 s}\right)$ be an admissible boundary datum and let $\psi_{a}$ be the solution to (3.2) with boundary datum $\Phi^{a}=\sum_{j=1}^{2 s}(-1)^{j} \phi_{j}$. The point $p \in D$ is a 2 s-point for the function $U=\left|\psi_{a}\right|$ if and only if $\Phi^{a}$ satisfies conditions (3.13).

\section{Results on 6 species}

In this section we consider the case of 6 competing species. As a consequence of Proposition 3.2 we show that 5 limiting configurations are possible.

Proposition 4.1 Let $U \in \mathcal{S}_{\Phi}$, then only one of the following statement is satisfied

i. $Z_{3}(U)$ consists of one point $a_{U} \in \bar{D}$ such that $m\left(a_{U}\right)=6$,

ii. $Z_{3}(U)$ consists of two points $a_{U}, b_{U} \in \bar{D}, a_{U} \neq b_{U}$, with $m\left(a_{U}\right)=m\left(b_{U}\right)=4$,

iii. $Z_{3}(U)$ consists of two points $a_{U}, b_{U} \in \bar{D}$ with $m\left(a_{U}\right)=3$ and $m\left(b_{U}\right)=5$,

iv. $Z_{3}(U)$ consists of three different points $a_{U}, b_{U}, c_{U} \in \bar{D}$ with $m\left(a_{U}\right)=4, m\left(b_{U}\right)=m\left(c_{U}\right)=$ 3 ,

v. $Z_{3}(U)$ consists of four different points $a_{U}, b_{U}, c_{U}, d_{U} \in \bar{D}$ with $m\left(a_{U}\right)=m\left(b_{U}\right)=$ $m\left(c_{U}\right)=m\left(d_{U}\right)=3$.

Proof. The set $Z_{3}(U)$ is nonempty and contains at most 4 points. If $Z_{3}(U)=\left\{a_{U}\right\}$ then, from (3.1), we deduce that $m\left(a_{U}\right)=6$ (cf. Figure 2). If $Z_{3}(U)=\left\{a_{U}, b_{U}\right\}$ then, from (3.1),
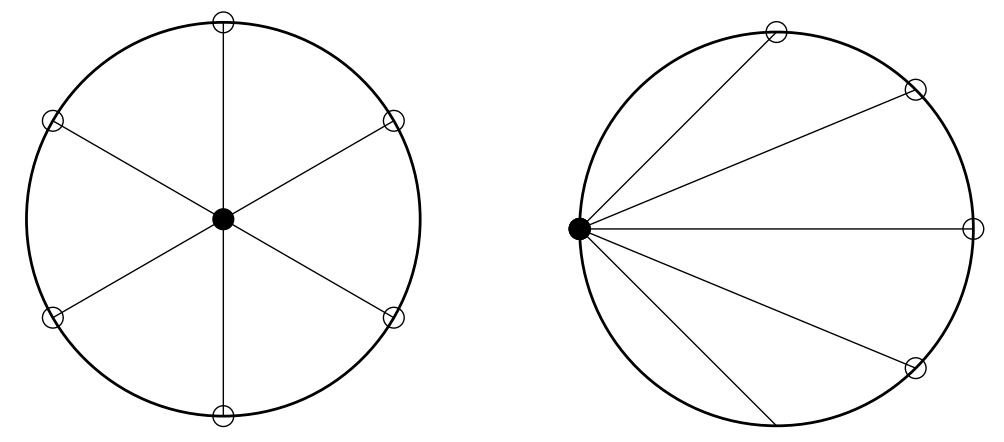

FIG. 2. Configurations with one 6-point inside $D$ (on the left) and on $\partial D$ (on the right) 

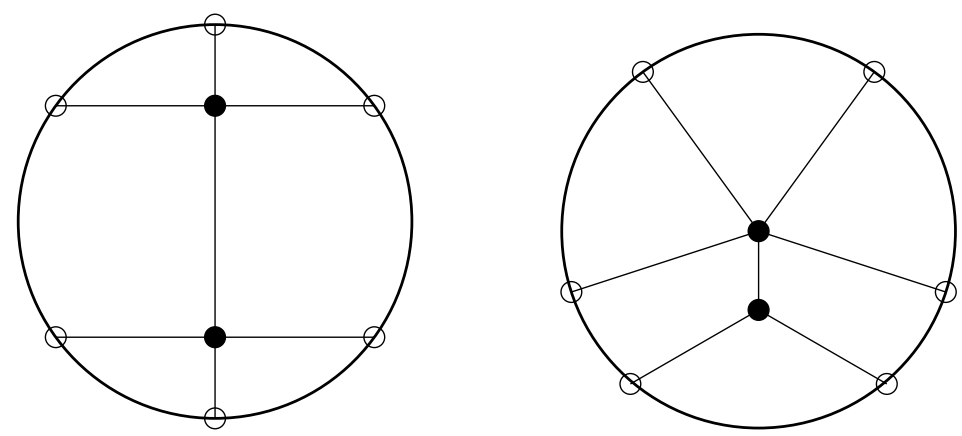

FIG. 3. Configurations when $Z_{3}(U)$ consists of two points $a_{U} \neq b_{U}$, with $m\left(a_{U}\right)=m\left(b_{U}\right)=4$ (on the left) and $m\left(a_{U}\right)=3$ and $m\left(b_{U}\right)=5$ (on the right)
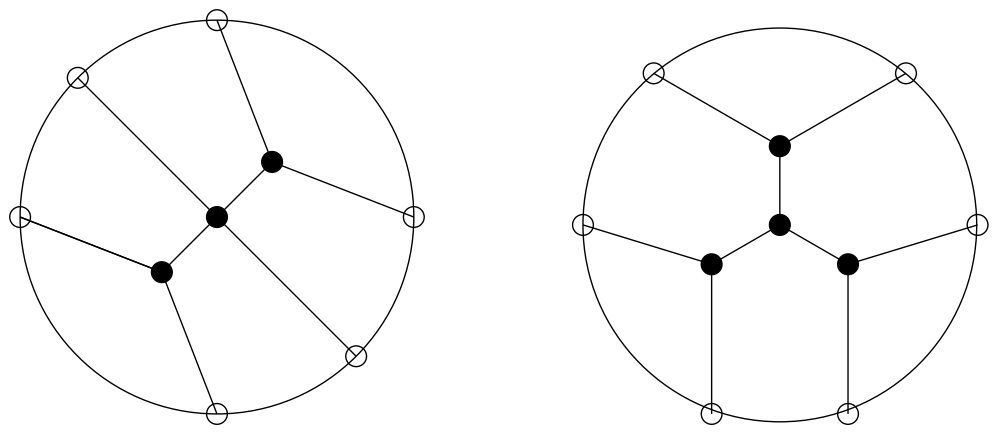

FIG. 4. Configurations when $Z_{3}(U)$ consists of three points (on the left) and four points (on the right)

we deduce that $m\left(a_{U}\right)+m\left(b_{U}\right)=8$, that is ii. or iii. (cf. Figure 3). If $Z_{3}(U)=\left\{a_{U}, b_{U}, c_{U}\right\}$ then, from (3.1), we deduce that $m\left(a_{U}\right)+m\left(b_{U}\right)+m\left(c_{U}\right)=10$, that is iv. (cf. Figure 4 on the left). If $Z_{3}(U)=\left\{a_{U}, b_{U}, c_{U}, d_{U}\right\}$ then, from (3.1), we deduce that $m\left(a_{U}\right)+m\left(b_{U}\right)+m\left(c_{U}\right)+m\left(d_{U}\right)=$ 12 , that is v. (cf. Figure 4 on the right).

In the case of 6 species, Theorem 3.13 can be formulated as follows.

Proposition 4.2 Let $\Phi=\left(\phi_{1}, \ldots, \phi_{6}\right)$ be an admissible datum, let $U \in S_{\Phi}$ and let $\psi_{a}$ be the solution to (3.2) with boundary datum $\Phi^{a}=\sum_{j=1}^{6}(-1)^{j} \phi_{j}$. The function $U$ has a 6 -point $p \in D$ if and only if the boundary datum $\Phi=\left(\phi_{1}, \ldots, \phi_{6}\right)$ satisfies the conditions

$$
\begin{aligned}
& \sum_{j=1}^{6}(-1)^{j} \int_{\partial D} \phi_{j}\left(\frac{\zeta+p}{\bar{p} \zeta+1}\right) d s_{\zeta}=0, \\
& \sum_{j=1}^{6}(-1)^{j} \int_{\partial D} \phi_{j}\left(\frac{\zeta+p}{\bar{p} \zeta+1}\right) \zeta_{r} d s_{\zeta}=0, \quad r=1,2 \\
& \sum_{j=1}^{6}(-1)^{j} \int_{\partial D} \phi_{j}\left(\frac{\zeta+p}{\bar{p} \zeta+1}\right) \zeta_{1}^{2} d s_{\zeta}=0,
\end{aligned}
$$




$$
\sum_{j=1}^{6}(-1)^{j} \int_{\partial D} \phi_{j}\left(\frac{\zeta+p}{\bar{p} \zeta+1}\right) \zeta_{1} \zeta_{2} d s_{\zeta}=0
$$

Moreover, the function $U=\left|\psi_{a}\right|$.

Proof. Conditions (4.1)-(4.4) are easily obtained by assuming $s=3$ in (3.13).

Proposition 4.3 Let $\Phi$ be an admissible datum and let $\psi_{a}$ be the solution to (3.2) with boundary datum $\Phi^{a}=\sum_{j=1}^{6}(-1)^{j} \phi_{j}$. Conditions (4.1)-(4.4) are equivalent to

$$
\psi_{a}(p)=0, \quad \nabla \psi_{a}(p)=(0,0), \quad H \psi_{a}(p)=\mathbf{0}
$$

where $H \psi_{a}=\left\{\partial_{x_{i} x_{j}}^{2} \psi_{a}\right\}_{i, j=1,2}$ denotes the Hessian matrix of the function $\psi_{a}$.

Proof. We introduce the transformation (3.6) and define $\widetilde{\Psi}_{a}$ and $\widetilde{\Phi}^{a}$ according to (3.7). We have

$$
\begin{gathered}
\psi_{a}(p)=\psi_{a}\left(R_{p}(0)\right)=\widetilde{\Psi}_{a}(0) \\
\nabla_{x} \psi_{a}(p)=\left(\begin{array}{cc}
\left(1-|p|^{2}\right)^{-1} & 0 \\
0 & \left(1-|p|^{2}\right)^{-1}
\end{array}\right) \nabla_{\zeta} \widetilde{\Psi}_{a}(0)
\end{gathered}
$$

and, assuming $p=\left(p_{1}, p_{2}\right)$,

$$
\begin{aligned}
H_{x} \psi_{a}(p)=\frac{1}{\left(1-|p|^{2}\right)^{2}} H_{\zeta} \widetilde{\Psi}_{a}(0) & \\
& +\frac{2}{1-|p|^{2}}\left(\partial_{\zeta_{1}} \widetilde{\Psi}_{a}(0)\left(\begin{array}{cc}
p_{1} & p_{2} \\
p_{2} & -p_{1}
\end{array}\right)+\partial_{\zeta_{2}} \widetilde{\Psi}_{a}(0)\left(\begin{array}{cc}
-p_{2} & p_{1} \\
p_{1} & p_{2}
\end{array}\right)\right)
\end{aligned}
$$

By construction, $\widetilde{\Psi}_{a}$ solves (3.8). By the Poisson integral formula (3.12) we deduce that

$$
\widetilde{\Psi}_{a}(0)=\frac{1}{2 \pi} \int_{\partial D} \frac{\widetilde{\Phi}^{a}(\eta)}{|\eta|^{2}} d s_{\eta}=\frac{1}{2 \pi} \int_{\partial D} \widetilde{\Phi}^{a}(\eta) d s_{\eta}=\frac{1}{2 \pi} \int_{\partial D} \Phi^{a}\left(R_{p}(\eta)\right) d s_{\eta} .
$$

On the other hand, by direct differentiation of the Poisson integral, we get

$$
\begin{gathered}
\frac{\partial}{\partial \zeta_{j}} \widetilde{\Psi}_{a}(0)=\frac{1}{\pi} \int_{\partial D} \widetilde{\Phi}^{a}(\eta) \eta_{j} d s_{\eta}=\frac{1}{\pi} \int_{\partial D} \Phi^{a}\left(R_{p}(\eta)\right) \eta_{j} d s_{\eta}, \quad j=1,2, \\
\frac{\partial^{2}}{\partial^{2} \zeta_{j}} \widetilde{\Psi}_{a}(0)=-\frac{2}{\pi} \int_{\partial D} \widetilde{\Phi}^{a}(\eta) d s_{\eta}+\frac{4}{\pi} \int_{\partial D} \widetilde{\Phi}^{a}(\eta) \eta_{j}^{2} d s_{\eta}, \quad j=1,2 \\
\frac{\partial^{2}}{\partial \zeta_{1} \partial \zeta_{2}} \widetilde{\Psi}_{a}(0)=\frac{4}{\pi} \int_{\partial D} \widetilde{\Phi}^{a}(\eta) \eta_{1} \eta_{2} d s_{\eta} .
\end{gathered}
$$

The equivalence between (4.1)-(4.4) and (4.5) easily follows.

Proposition 4.4 Let $\Phi=\left(\phi_{1}, \ldots, \phi_{6}\right)$ be an admissible datum and suppose that the related harmonic function $\psi_{a}$, solution to (3.2) with boundary datum $\phi^{a}$, has two critical points $p, q \in D$ such that $\psi_{a}(p)=\psi_{a}(q)=0$, then $p, q$ are 4-points for the function $U=\left|\psi_{a}\right| \in \mathcal{S}_{\Phi}$. 
Proof. By standard theory of harmonic functions the zero set of $\psi_{a}$ around a critical point at level 0 is made by (at least) 4 half-lines, meeting with equal angles. We infer that locally around $p$ the function $\psi_{a}$ defines $k_{p}$ nodal components with $k_{p} \geqslant 4$ and $k_{p}$ is even because $\psi_{a}$ has alternate positive or negative sign on adjacent sets. Analogously around $q$ the function $\psi_{a}$ defines $k_{q}$ nodal components with $k_{q} \geqslant 4$ and $k_{q}$ is even. By Proposition 4.1 we infer that $k_{p}=k_{q}=4$. Hence the function $U=\left|\psi_{a}\right|$ is nonnegative, satisfies the boundary datum $\Phi$, has exactly 6 nodal regions and generates an element of $\delta_{\Phi}$, with $p, q \in Z_{3}(U)$.

Conversely, as a direct application of Proposition 3.9 we have the following result.

Proposition 4.5 Let $\Phi=\left(\phi_{1}, \ldots, \phi_{6}\right)$ be an admissible datum. Let $U \in S_{\Phi}$ such that $Z_{3}(U)$ contains two points $p, q$ in $D$ with multiplicity 4. Then $U=\left|\psi_{a}\right|$, where $\psi_{a}$ is the harmonic function such that $\psi_{a}=\Phi^{a}=\sum_{j=1}^{6}(-1)^{j} \phi_{j}$ on $\partial D$. Moreover, $p, q$ are critical points for $\psi_{a}$ at zero level.

\section{Conclusion and open problems}

Propositions 4.4 and 4.5 show that the limiting configurations, which elements of $Z_{3}(U)$ have only even multiplicity, are closely connected to harmonic solutions of (3.2) with alternate boundary datum. Since such solutions have to satisfy some integral conditions (see (3.13)), it follows that the most probable segregation configurations have only points in $Z_{3}(U)$ with odd multiplicity (see also Remark 3.13 in [19]).

In fact, we think that the most probable configurations in nature are those with points with multiplicity three. But this means that odd multiple points (with multiplicity greater than 3 ) have to verify some conditions in order to belong to subsets of admissible data with nontrivial codimension. Now this is an open problem, since our techniques are strongly related to the properties of the harmonic functions, and these functions are related only to critical points with even multiplicity.

An open problem we wish to handle is to compare results by reaction-diffusion systems in populations competition, as in [3, 4, 9, 11, 12, 19, 23], and results obtained by differential games theory contained in $[16,20]$ (see also the references therein), in order to reach a better understanding of the territoriality of the competing species or groups (see also [5]).

Acknowledgment. The authors are very grateful to the anonymous referees for their insightful comments and helpful suggestions.

\section{References}

1. Abramowitz, M. \& Stegun, I., Handbook of Mathematical Functions. Dover Publ. 1970.

2. Arakelyan, V. \& Bozorgnia, F., Uniqueness of limiting solution to a strongly competing system. Elect. J. Diff. Eqs. 2017 (2017), Paper No. 96. Zbl1370. 35114 MR3651893

3. Berestycki, H. \& Zilio, A., Predators-prey models with competition, Part I: Existence, bifurcation and qualitative properties. Commun. Contemp. Math. 20 (2018), 1850010, 53. Zbl1404. 35452 MR3864295

4. Berestycki, H. \& Zilio, A., Predators-prey models with competition: The emergence of packs and territoriality, The American Naturalist 193 (2019), 436-446.

5. Börger, L., Dalziel, B. D. \& Fryxell, J. M., Are there general mechanisms of animal home range behaviour? A review and prospects for future research, Ecol. Lett. 11 (2008), 637-650. 
6. Cicalese, M., De Luca, L., Novaga, M., \& Ponsiglione, M., Ground states of a two phase model with cross and self attractive interactions. SIAM J. Math. Anal. 48 (2016), 3412-3443. Zbl1352 . 49009 MR3549880

7. Cody, W. J., A survey of practical rational and polynomial approximation of functions. SIAM Rev. 12 (1970), 400-423. Zb10199. 39301 MR267725

8. Conti, M., Terracini, S., \& Verzini, G., Nehari's problem and competing species systems. Ann. Inst. H. Poincaré Anal. Non Linéaire 19 (2002), 871-888. Zbl1090.35076 MR1939088

9. Conti, M., Terracini, S., \& Verzini, G., Asymptotic estimates for the spatial segregation of competitive systems. Adv. Math. 195 (2005), 524-560. Zbl1126. 35016 MR2146353

10. Conti, M., Terracini, S., \& Verzini, G., A variational problem for the spatial segregation of reactiondiffusion systems. Indiana Univ. Math. J. 54 (2005), 779-815. Zbl1132. 35397 MR2151234

11. Conti, M., Terracini, S., \& Verzini, G., Uniqueness and least energy property for solutions to strongly competing systems. Interfaces Free Bound. 8 (2006), 437-446. Zbl1103. 92041 MR2283921

12. Dancer, E. N., Wang, K., \& Zhang, Z., Dynamics of strongly competing systems with many species. Trans. Amer. Math. Soc. 364 (2012), 961-1005. Zbl1252.35284 MR2846360

13. Edelstein-Keshet, L., Mathematical Models in Biology. Vol. 46 of Classics in Applied Mathematics. Society for Industrial and Applied Mathematics (SIAM), Philadelphia, PA, 2005. Reprint of the 1988 original. MR2131632

14. Gause, G. F., Experimental studies on the struggle for existence: 1. Mixed population of two species of yeast. J. Exper. Biol. 9 (1932), 389-402.

15. Gilbarg, D. \& Trudinger, N. S., Elliptic Partial Differential Equations of Second Order. Classics in Mathematics. Springer-Verlag, Berlin, 2001. Reprint of the 1998 edition. Zbl1042. 35002 MR1814364

16. Hamelin, F. M. \& Lewis, M. A., A differential game theoretical analysis of mechanistic models for territoriality. J. Math. Biol. 61 (2010), 665-694. Zb11205. 92075 MR2684159

17. Hardin, G., The competive exclusion principle. Science, New Series 131 (1960), 1292-1297.

18. Jungnickel, D., Graphs, Networks and Algorithms. Third ed., vol. 5 of Algorithms and Computation in Mathematics. Springer, Berlin, 2008. MR2363884

19. Lanzara, F., \& Montefusco, E., On the limit configuration of four species strongly competing systems. NoDEA Nonlinear Differential Equations Appl. 26 (2019), Paper No. 19, 17. Zb11421. 35154 MR3948936

20. Morrell, L. J. \& Kokko, H., Bridging the gap between mechanistic and adaptive explanations of territory formation. Behav. Ecol. Sociobiol. 57 (2005), 381-390.

21. Ponce, A. C., Elliptic PDEs, Measures and Capacities. From the Poisson Equations to Nonlinear ThomasFermi Problems. Vol. 23 of EMS Tracts in Mathematics. European Mathematical Society (EMS), Zürich, 2016. Zbl1357. 35003 MR3675703

22. Tavares, H. \& Terracini, S., Regularity of the nodal set of segregated critical configurations under a weak reflection law. Calc. Var. Partial Differential Equations 45 (2012), 273-317. Zbl1263. 35101 MR2984134

23. Terracini, S., Verzini, G., \& Zilio, A., Spiraling asymptotic profiles of competition-diffusion systems. Comm. Pure Appl. Math. 72 (2019), 2578-2620. Zbl1433. 35141 MR4020313

24. Wang, K. \& Zhang, Z., Some new results in competing systems with many species. Ann. Inst. H. Poincaré Anal. Non Linéaire 27 (2010), 739-761. Zbl1201. 35113 MR2595199 